\title{
Long-Term Effects of Cannabis on Brain Structure
}

\author{
Giovanni Battistella $^{1,8}$, Eleonora Fornari ${ }^{1,2,8}$, Jean-Marie Annoni ${ }^{3}$, Haithem Chtioui ${ }^{4}, \mathrm{Kim} \mathrm{Dao}^{4}$, \\ Marie Fabritius ${ }^{5}$, Bernard Favrat ${ }^{6}$, Jean-Frédéric Mall ${ }^{7}$, Philippe Maeder*, ${ }^{*, 8}$ and Christian Giroud ${ }^{5,8}$ \\ 'Department of Radiology, Centre Hospitalier Universitaire Vaudois (CHUV), University of Lausanne, Lausanne, Switzerland; ${ }^{2}$ CIBM (Centre \\ d'Imagerie Biomédicale), Centre Hospitalier Universitaire Vaudois (CHUV) unit, Lausanne, Switzerland; ${ }^{3}$ Neurology Units, Department of \\ Medicine, University of Fribourg, Fribourg, Switzerland; ${ }^{4}$ Department of Clinical Pharmacology and Toxicology, Centre Hospitalier Universitaire \\ Vaudois CHUV, Lausanne, Switzerland; ${ }^{5}$ CURML (University Center of Legal Medicine), UTCF (Forensic Toxicology and Chemistry Unit), \\ Lausanne, Switzerland; ' CURML (University Center of Legal Medicine), UMPT (Unit of Psychology and Traffic Medicine), Lausanne and Geneva, \\ Switzerland; 'Department of Psychiatry, SUPAA (Service Universitaire de Psychiatrie de l'Age Avancé), CHUV, Lausanne, Switzerland
}

\begin{abstract}
The dose-dependent toxicity of the main psychoactive component of cannabis in brain regions rich in cannabinoid CBI receptors is well known in animal studies. However, research in humans does not show common findings across studies regarding the brain regions that are affected after long-term exposure to cannabis. In the present study, we investigate (using Voxel-based Morphometry) gray matter changes in a group of regular cannabis smokers in comparison with a group of occasional smokers matched by the years of cannabis use. We provide evidence that regular cannabis use is associated with gray matter volume reduction in the medial temporal cortex, temporal pole, parahippocampal gyrus, insula, and orbitofrontal cortex; these regions are rich in cannabinoid CBI receptors and functionally associated with motivational, emotional, and affective processing. Furthermore, these changes correlate with the frequency of cannabis use in the 3 months before inclusion in the study. The age of onset of drug use also influences the magnitude of these changes. Significant gray matter volume reduction could result either from heavy consumption unrelated to the age of onset or instead from recreational cannabis use initiated at an adolescent age. In contrast, the larger gray matter volume detected in the cerebellum of regular smokers without any correlation with the monthly consumption of cannabis may be related to developmental (ontogenic) processes that occur in adolescence.

Neuropsychopharmacology (2014) 39, 204I-2048; doi:I0.1038/npp.20 I4.67; published online I6 April 20I4
\end{abstract}

\section{INTRODUCTION}

Cannabis is one of the most widely used recreational drugs, taking third place among drugs of concern in addiction treatment services (Degenhardt et al, 2008). Despite these statistics pointing to the potential harms associated with longterm cannabis use, little is known about the progression from recreational to regular use and its effects on brain structure.

Current knowledge is mostly inferred from animal studies; it has been demonstrated that the main psychoactive component of cannabis $(\Delta 9$-Tetrahydrocannabinol, THC) induces dose-dependent toxicity and structural changes in brain regions rich in cannabinoid CB1 receptors. These are mainly located in the hippocampus, amygdala, cerebellum, prefrontal cortex, and striatum (Burns et al, 2007; Downer et al, 2001; Lawston et al, 2000).

In contrast to the animal literature, the investigation of the structural effects of long-term cannabis use on the human brain has brought less consistent findings. Changes in gray or white matter density have been reported in

*Correspondence: Professor P Maeder, Department of Radiology, CHUV, Rue du Bugnon 46, Lausanne CH-IOII, Switzerland, Tel: +4I 795561684, Fax: +41 213144554,

E-mail: Philippe.Maeder@chuv.ch

${ }^{8}$ These authors contributed equally to this work.

Received 5 December 2013; revised 22 February 2014; accepted 6

March 20I4; accepted article preview online 17 March 2014 different locations in frontal and parietal lobes without overlapping findings across studies (Churchwell et al, 2010; Gruber et al, 2011; Matochik et al, 2005). The discrepancy in the results might be due to heterogeneity in sample characteristics, inter-individual differences linked to past history of drug use, amount of consumption, related psychological problems (temperament, level of anxiety or arousal), and/or methodological differences in data processing (Batalla et al, 2013; Lorenzetti et al, 2010). However, changes in the hippocampus/parahippocampal complex and in the amygdala have often been reported (Demirakca et al, 2011; Matochik et al, 2005; Yücel et al, 2008; Zalesky et al, 2012). These findings suggest that long-term cannabis use is associated with brain morphology alterations in regions linked to memory and executive and affective processing (Yücel et al, 2008).

Decrease in hippocampal volume in regular cannabis smokers has been correlated with lifetime consumption (Ashtari et al, 2011; Yücel et al, 2008) and psychotic symptoms (Yücel et al, 2008). In Cousijn et al (2012), volume reduction in the amygdala and the hippocampus does not differ significantly between regular cannabis users and controls but still correlates with the amount of cannabis used and the severity of cannabis dependence, respectively.

In addition to the several issues characterizing the literature so far, most of the studies examine only predefined regions of interest (i.e., the hippocampus and 
amygdala because of their richness in CB1 receptors), making it difficult to draw consistent and complete conclusions about the long-term effects of cannabis use on brain structure.

Another question of interest is whether cannabis use is associated with differential effects on brain structure according to the age of consumption onset; more specifically, does the use of cannabis during adolescence lead to the same changes in brain structure as initial exposure later in life? Adolescence is an important period in brain development when proper structural maturation of fiber tracts occurs (necessary for the development of cognitive, motor, and sensory functions) (Paus et al, 1999). Environmental factors, such as drug use, can alter the maturational arrangements that normally occur in the adolescent brain (Hurd et al, 2013), increasing the incidence of psychiatric illness and substance abuse (Paus et al, 2008). The study by Zalesky et al (2012) shows a linear correlation between measures of white matter (WM) integrity and the age of onset of regular cannabis use, thus suggesting a toxic effect of long-term cannabis use to WM development.

In our study, we investigate the precise nature of structural changes induced by cannabis and try to answer three main questions: (i) Can we observe temporal and frontal (including insular) gray matter changes after long-term exposure to cannabis? (ii) Is the magnitude of these changes related to the amount of cannabis consumed? (iii) Are these changes influenced by the age of first use (during/after adolescence)?

Unlike many previous studies, we used a whole-brain voxel-wise approach in order to localize gray matter change due to long-term exposure to cannabis. To this end, we used Voxel-based Morphometry (VBM), a completely automatic and unbiased data processing technique for the assessment of gray matter density. This technique has been already widely used in clinical research (Draganski and Bhatia, 2010) and in drug addiction (Connolly et al, 2013).

Brain structure changes were investigated in a group of regular cannabis smokers and compared with a group of occasional smokers enrolled in our previous functional study (Battistella et al, 2013). The subjects in the two groups did not use any drug other than cannabis and were free from psychiatric disorders. We then stratified the two groups according to the age of first cannabis use in order to assess the effect of cannabis on the developing brain.

\section{MATERIALS AND METHODS}

The present study is a part of a Magnetic Resonance Imaging (MRI) study with the aim of investigating (at the functional and structural levels) the acute and chronic effects of cannabis smoking on specific skills related to driving ability (Battistella et al, 2013). The study was conducted according to Good Clinical Practice and ICH requirements and has been approved by the local Ethics Committee of the Canton de Vaud (Lausanne) Switzerland.

\section{Subjects and Recruitment}

Thirty-one healthy male occasional cannabis smokers between 18 and 30 years of age and twenty-six male regular cannabis users participated in the study. Subjects were recruited through public advertisements at universities. The mean consumption of cannabis for the 3 months preceding inclusion in the study was set to a minimum of one joint per month and a maximum of less than one joint per week for the occasional smokers, and to a minimum of 10 joints per month for regular ones (Fabritius et al, 2013a). All volunteers had no history of neurological or psychiatric disorders. Nine occasional and one regular users were excluded from the research protocol for different reasons (claustrophobia, detection of brain anomalies, decision to drop out of the study, or technical problems during data processing). The final population selected for this study consists of 22 occasional cannabis smokers (median age 25 years, SD 2.8 years) and 25 regular users (median age 23 years, SD 2.2 years).

All participants were carefully screened with an interview and a medical evaluation to ensure that they fulfilled all study criteria. Further details regarding the inclusion procedure are indicated in Battistella et al (2013). In both groups of cannabis users, we performed a urine and a blood test during the inclusion process in order to determine the concentration of $\Delta 9$-Tetrahydrocannabinol-carboxylic acid $(\mathrm{THCCOOH})$ and confirm the regular use and the extent of cannabis consumption (Fabritius et al, 2013b). Participants also filled out a questionnaire about their usual drug use habits. Cannabinoid time profiles in whole blood were determined with the analytical procedures described by Fabritius et al (2013a). Presence of other illicit drugs (cocaine, opiates, and amphetamines) and a positive breath alcohol test were also considered as exclusion criteria.

Major characteristics of the population are shown in Table 1; for each variable of interest, we tested the null hypothesis of whether the data followed a normal distribution using the Shapiro-Wilk test. As the null hypothesis was rejected, we performed group comparisons by the mean of the non-parametric Wilcoxon-signed ranks test.

\section{MRI Acquisition Protocol}

Scanning was performed on a $3 \mathrm{~T}$ Siemens Trio scanner equipped with a 32-channel head coil. The protocol included a high-resolution sagittal T1-weighted 3D gradient-echo sequence (MPRAGE), 160 contiguous slices, $1 \mathrm{~mm}$ isotropic voxel, repetition time (TR) $2300 \mathrm{~ms}$, echo time (TE) $2.98 \mathrm{~ms}$, field of view $256 \mathrm{~mm}$. We prevented head movements by cushioning the participant's head in the coil with padding.

\section{MRI Data Processing}

MRI data were pre-processed and analyzed using Statistical Parametric Mapping (SPM8, Wellcome Department of Cognitive Neurology, London, UK). For Voxel-Based morphometry (VBM) analysis, T1-weighted data were classified as gray matter, white matter (WM), and cerebrospinal fluid (CSF) using the unified segmentation approach (Ashburner and Friston, 2005). In order to improve registration accuracy, images were non-linearly transformed to the standard Montreal Neurological Institute (MNI) space using the diffeomorphic registration algorithm (DARTEL) (Ashburner, 2007). After registration and bias correction, GM probability maps were subsequently 
Table I Descriptive Statistics of the Subjects Enrolled in the Study

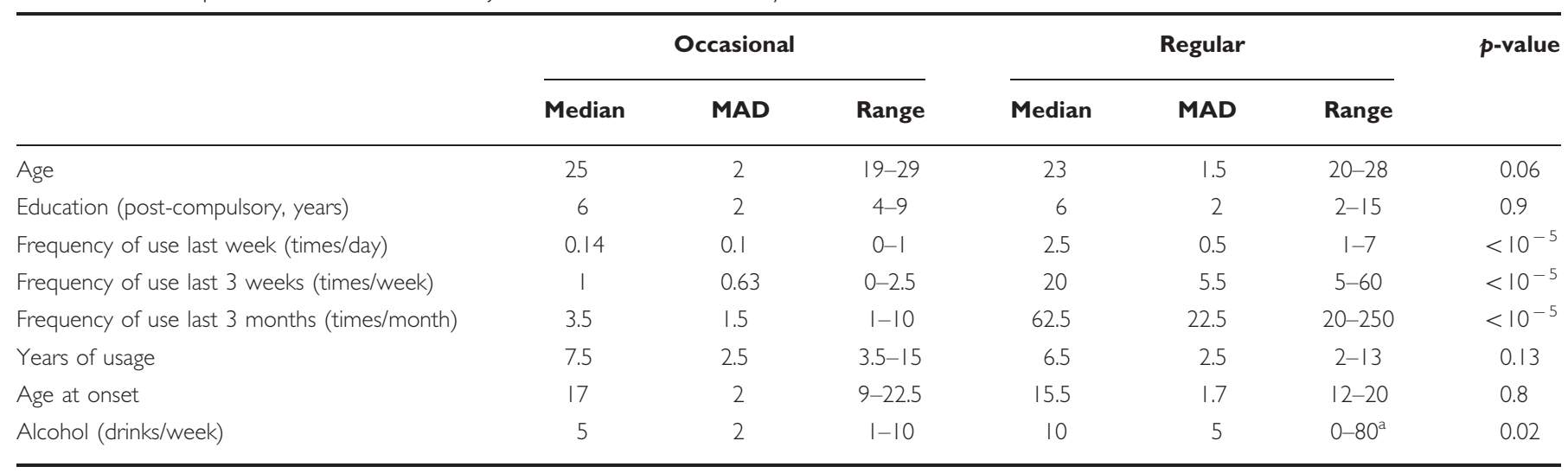

We report median values, median absolute deviation (MAD), range and significance of the between groups' comparison by mean of Wilcoxon-signed rank test. The years of usage and the age of onset refer to any cannabis use, and the alcohol consumption refers to drinks of $10 \mathrm{~g}$ ethanol.

${ }^{a}$ Only one subject declared a weekly alcohol consumption of 80 drinks/week (outlier), all the other subjects ranged between 0 and 20.

'modulated' by the Jacobian determinants of the deformations to account for local compression and expansion during linear and non-linear transformation (Good et al, 2001). Finally, GM probability maps were smoothed with an isotropic Gaussian kernel of $6 \mathrm{~mm}$ full width at half maximum (FWHM) to improve Signal-to-Noise Ratio (SNR) and to conform the data more closely to a Gaussian distribution for statistics. Voxel-based inferential statistics were performed on the smoothed modulated gray matter images using a two-sample $t$-test embedded in the Random Effect framework of SPM8. We included age, total brain volume (TBV, the sum of gray matter and white matter), and alcohol consumption as regressors of no interest to control for the effects of these variables. Statistical thresholds were applied at $p<0.05$ after family-wise error (FWE) correction for multiple comparisons over the whole brain and $k>60$ for cluster extent (greater than the minimum voxels expected per cluster). Voxel-by-voxel correlation analysis was performed between the smoothed modulated gray matter images and the variable representative of the level of drug use (monthly frequency of joints used in the 3 months before inclusion in the study) after testing the presence of outliers.

Then, each group (occasional/regular) was stratified according to the age of first use (before/after 18 years of age) in order to assess whether the age at which cannabis use started influences the magnitude of the decreased gray matter volume localized with the two-sample $t$-test. The four subgroups were composed of 13 occasional smokers who started cannabis consumption before the age of 18 (called here 'Occasional early'), and 9 who started after (called 'Occasional late'); 16 regular users who started cannabis consumption before the age of 18 (called here 'Regular early'), and 9 who started after (called 'Regular late'). Gray matter volume, represented by the value of the modulated GM image, was compared between the so-defined subgroups. We tested significant differences between the 'Occasional late' group and all of the other groups (ie, three tests for each region) at a level of $p<0.05$ (corrected for multiple comparisons). For each comparison between subgroups, we also computed the effect size (d) by dividing the difference between mean values by the common SD of the two groups (Cohen, 1988).

\section{RESULTS}

Self-rating questionnaires show a significant difference in the frequency and amount of cannabis consumption between the groups of regular and occasional smokers. However, they do not differ in age, in the years of cannabis use and or in the age at which consumption started (Table 1). The median value of the self-reported usual amount of cannabis smoked by regular users is higher than that of occasional consumers $(0.4 \mathrm{~g} v s 0.3 \mathrm{~g})$. The determination of cannabinoid time profiles revealed that the THCCOOH median level was significantly higher in regular smokers compared with occasional users $(21 \mu \mathrm{g} / \mathrm{l} v s 0 \mu \mathrm{g} / \mathrm{l}$ just before smoking the joint). An equal difference was found for the participants enrolled in the same study and selected by Fabritius et al, (2013a) for the pharmacokinetic determinations.

\section{MRI Results}

When comparing gray matter volume between groups, we find that significant clusters showing a lower gray matter volume in regular cannabis users compared with occasional ones are located bilaterally in the temporal pole and in the parahippocampal gyrus. Additional clusters cover the left insula and the left orbitofrontal cortex (Figure 1). In contrast, three cerebellar clusters show the opposite behavior, with increased gray matter volume. Coordinates of the centers of gravity of the significant clusters are reported in the Montreal Neurological Institute (MNI) space and are summarized in Tables 2 and 3.

Voxel-by-voxel correlations over the whole brain were performed merging the two groups together. Correlation analysis highlights an inverse linear correlation between GM volume and the monthly frequency of cannabis use during the 3 months before inclusion in the study. Regions with a decreased GM volume in regular smokers (Table 2) are 


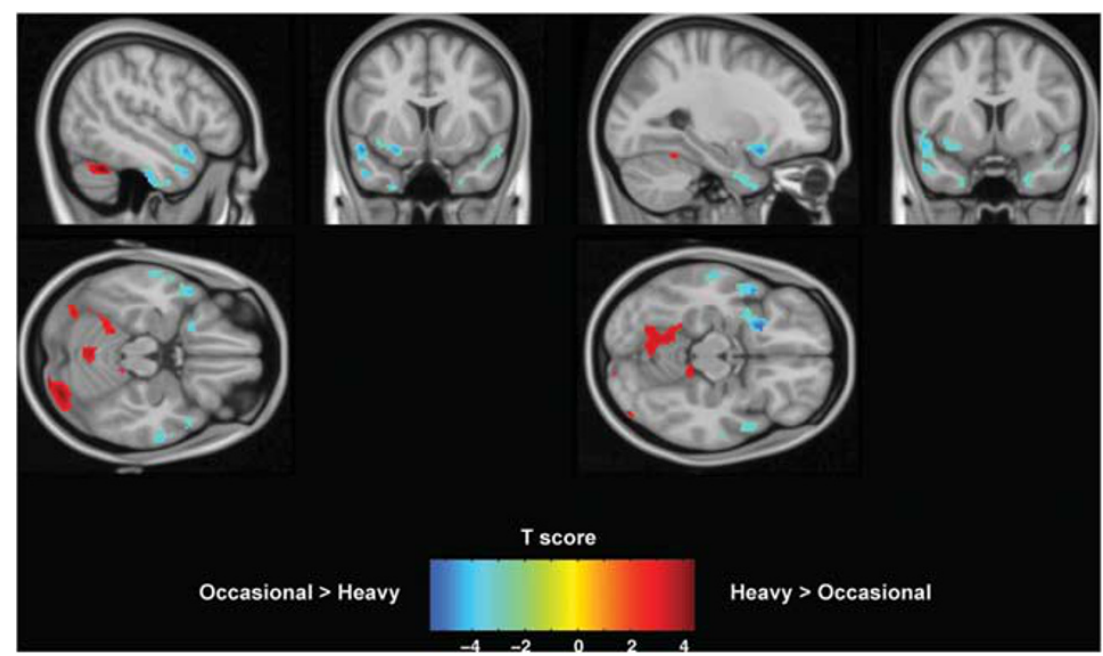

Figure I Voxel-Based Morphometry results on gray matter. Cold color bar shows regions where gray matter volume is lower in regular smokers compared with occasional ones. Hot color bar represents the opposite contrast. Maps are thresholded at $P<0.005$ and $k>60$ and superposed on a standard brain in the MNI space. Figure shows results in planes centered at $-26,7,14 \mathrm{~mm}$ and $-48,10,-19 \mathrm{~mm}$. Color bars represent T score.

Table 2 Local Maxima of Significant Clusters Showing Decreased Gray Matter Volume in Regular Cannabis Smokers

\begin{tabular}{|c|c|c|c|c|c|c|c|c|c|c|}
\hline \multirow[t]{2}{*}{ Region } & \multicolumn{3}{|c|}{$\begin{array}{l}\text { Left hemisphere MNI } \\
\text { coordinates }(\mathrm{mm})\end{array}$} & \multirow[t]{2}{*}{$T$-value } & \multirow[t]{2}{*}{ Cluster extent } & \multicolumn{3}{|c|}{$\begin{array}{l}\text { Right hemisphere MNI } \\
\text { coordinates }(\mathrm{mm})\end{array}$} & \multirow[t]{2}{*}{$T$-value } & \multirow[t]{2}{*}{ Cluster extent } \\
\hline & $\mathbf{x}$ & $y$ & $\mathbf{z}$ & & & $\mathbf{x}$ & $y$ & $\mathbf{z}$ & & \\
\hline Superior orbital gyrus & -22 & 14 & -14 & 5.15 & & & & & & \\
\hline Temporal pole & -50 & 10 & -14 & 5.14 & & 50 & 14 & -20 & 3.59 & $213^{\mathrm{a}}$ \\
\hline Middle temporal gyrus & -56 & 2 & -28 & 4.84 & 1215 & 64 & -10 & -20 & 4.36 & $27 \mid$ \\
\hline Insula lobe & -36 & 8 & -10 & 4.22 & & & & & & \\
\hline Parahippocampal gyrus & -26 & 10 & -42 & 3.98 & 130 & 24 & 6 & -36 & 3.62 & 64 \\
\hline Precuneus & & & & & & 12 & -54 & 16 & 3.83 & 85 \\
\hline
\end{tabular}

Belong to the same cluster.

Table 3 Local Maxima of Significant Clusters Showing Increased Gray Matter Volume in Regular Cannabis Smokers

\begin{tabular}{|c|c|c|c|c|c|c|c|c|c|c|}
\hline \multirow[t]{2}{*}{ Region } & \multicolumn{3}{|c|}{$\begin{array}{l}\text { Left hemisphere MNI } \\
\text { coordinates }(\mathrm{mm})\end{array}$} & \multirow[t]{2}{*}{$T$-value } & \multirow[t]{2}{*}{ Cluster extent } & \multicolumn{3}{|c|}{$\begin{array}{l}\text { Right hemisphere MNI } \\
\text { coordinates }(\mathrm{mm})\end{array}$} & \multirow[t]{2}{*}{$T$-value } & \multirow[t]{2}{*}{ Cluster extent } \\
\hline & $\mathbf{x}$ & $y$ & $\mathbf{z}$ & & & $\mathbf{x}$ & $y$ & $\mathbf{z}$ & & \\
\hline Lobule VIla Crus I & -48 & -54 & -28 & 4.27 & 431 & 30 & -86 & -22 & 4.56 & 562 \\
\hline Lobule VI & -14 & -54 & -12 & 4 & 534 & & & & & \\
\hline
\end{tabular}

those that exhibit this inverse correlation $(P<0.005)$. Figure 2 (panel a) illustrates this relation in three clusters located in the left parahippocampal gyrus $(P=0.004, R=-0.42)$, left insula $(P=0.0002, \mathrm{R}=-0.54)$, and right temporal pole $(P=0.002, R=-0.45)$. The results for the other four regions are presented in the Supplementary File S1.

The stratification of the two groups according to the age of first cannabis use in the same clusters shows that a decrease in gray matter volume can occur with a heavy amount of cannabis consumption, independent of the years of usage (Figure 2, panel b). The comparison between the 'Occasional late' subgroup and both the 'Regular' subgroups shows a significant difference at $P<0.05$ in each cluster of interest. Recreational consumption begun early in adolescence (ie, 'Occasional early' subgroup) significantly affects the GM volume in two regions out of three. These are located in left parahippocampal gyrus $(P=0.04)$ and right temporal pole $(P=0.04)$; the left insula shows only a trend 

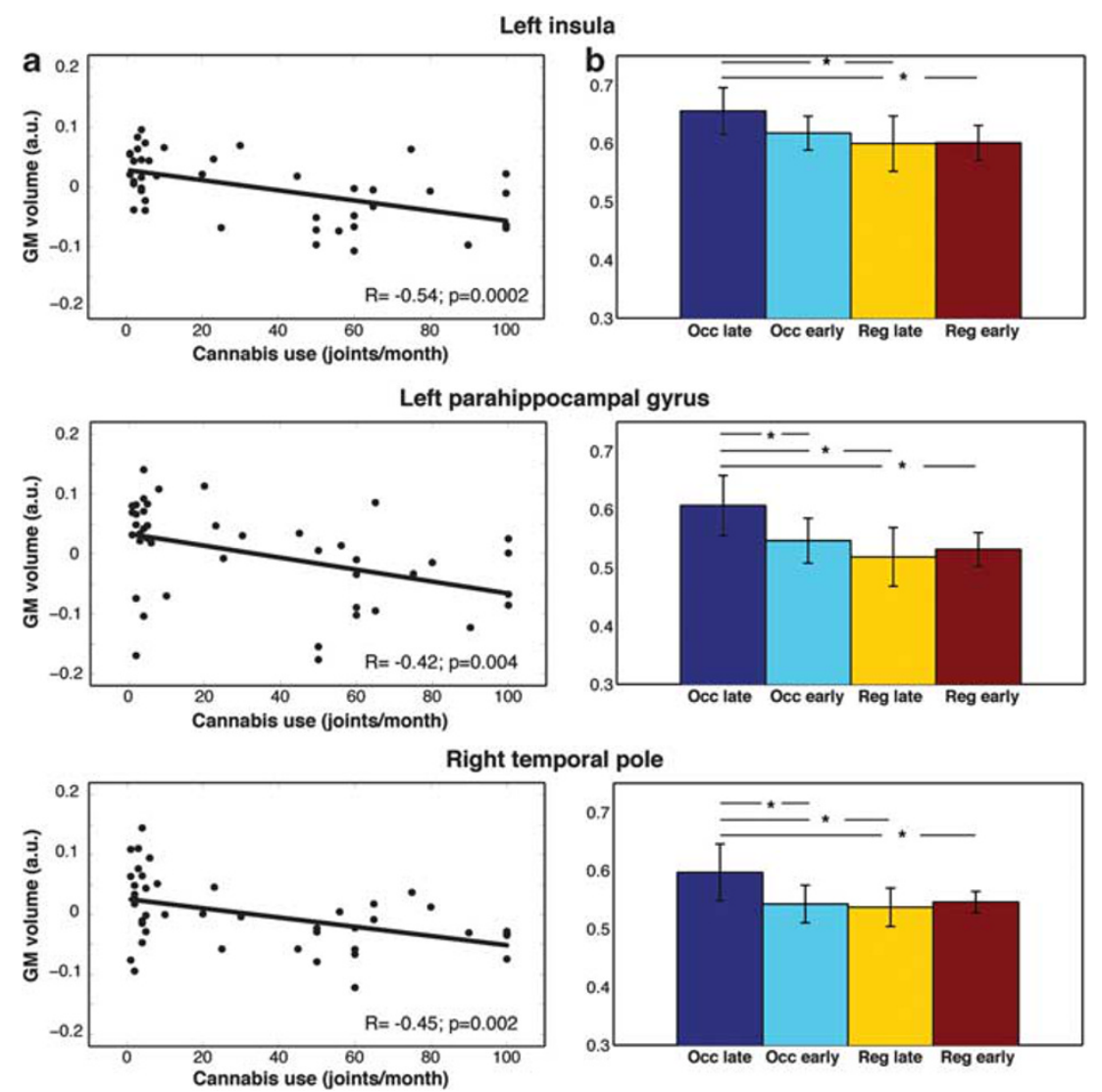

Figure 2 (a) Correlation between the modulated gray matter intensity at the center of gravity of the significant clusters and the monthly frequency of joints smoked during 3 months before inclusion in the study. Lines represent the fitting of the distribution of the values. Pearson's correlation coefficient and $P$-value are shown at the bottom of each plot. (b) Mean GM volume across the four subgroups (Occasional late, Occasional early, Regular late, Regular early). Whiskers represent $95 \%$ confidence interval, horizontal lines represent significant comparisons and stars the significance level $(P<0.05)$.

at $P=0.09$. The effect size measured by Cohen's $d$ was large $(d>1)$ in each significant comparison. The results for the other four regions are presented in the Supplementary File S1.

\section{DISCUSSION}

Our study demonstrates a pattern of gray matter volume changes in a group of regular cannabis users compared with a group of occasional ones. Regular users exhibit a decrease in gray matter (GM) volume in the medial temporal cortex, temporal pole, parahippocampal gyrus, left insula, and orbitofrontal cortex. These changes strongly correlate with the monthly frequency of cannabis use in the 3 months before inclusion in the study. We chose the preceding 3-month period as the period of interest because the participants' cannabis use during this length of time is representative of their usual cannabis use. Three clusters in the cerebellum show the opposite behavior, with increased GM volume. We also show that the age of onset of cannabis use is correlated with the magnitude of gray matter volume reduction in the cerebral hemispheres. Specifically, significant gray matter atrophy can occur either with a heavy cannabis consumption independent of the age of first use or with recreational consumption that started during adolescence (before the age of 18).

Our finding corroborates several animal studies (Burns et al, 2007; Downer et al, 2001; Lawston et al, 2000), adding evidence that the duration of exposure to cannabis is indeed associated with localized volume reduction in regions rich in CB1 receptors, correlating with the amount of cannabis used.

The progression of a long-term exposure to drugs toward the development of substance use disorders and addicted behaviors is often associated with deficits in decision making (Koob and Volkow, 2010; Wiers et al, 2007). FMRI demonstrates altered brain activity in core regions linked to the motivational and affective aspects of decision making (Cousijn et al, 2012; Vaidya et al, 2012), mainly in the ventromedial prefrontal (VMPFC) and orbitofrontal cortices and insula. In this regard, it has been demonstrated that substance-dependent individuals and patients with VMPFC lesions exhibit similar behaviors that lead them to make similar decisions in real life, preferring choices that bring immediate benefits even if coupled with negative consequences (Bechara and Damasio, 2002).

In our study, we complement the functional evidence of altered activity in nodes related to decision making by showing in regular cannabis users a decrease in gray matter volume in the insula, orbitofrontal cortex, and precuneus, regions that are part of the motivational and affective components of this network.

This observation supports and extends the conclusions about compromised activity in the Salience Network nodes (Seeley et al, 2007) described in occasional cannabis users following the smoking of a single joint of cannabis 
(Battistella et al, 2013). The key role in this network is played by the insula, a region involved in subjects' awareness of error, in the processing of affective and internal information and in switching between the different brain networks. Changes in this structure have also been confirmed in alcohol addiction where the decrease in insular activation seems to reflect an inability to switch from interoceptive cravings to cognitive control for suppressing internal needs (Sullivan et al, 2013). Here we complement this finding of functional change with its structural substrate. On the other hand, the activity in other nodes of the Salience and the Control Executive networks (VMPFC, ACC, Dorsolateral Prefrontal cortex) that is compromised in occasional smokers is not associated with structural changes in these regions in regular cannabis smokers. It remains thus to be explored whether morphological variations in these areas may occur at a later stage or in the presence of more serious addiction-related behaviors that our participants do not exhibit.

In relation to gray matter volume reduction in regions linked to affective and emotional processes described so far (the insula and the orbitofrontal cortex), we also show structural variations in the temporal pole (TP). The TP shares cytoarchitectural and functional characteristics with the orbitofrontal cortex (Kling and Steklis, 1976) and receives projections from the insula. The volume reduction in these regions observed in our population supports the idea of a joint role of these structures in regular cannabis use. Evidence exists about the role of the temporal pole (TP) in coupling emotions and highly processed sensory stimuli (Olson et al, 2007). Lesions in this structure lead to changes in personality and in social behavior (Thompson et al, 2003). Ablation of the monkey orbitofrontal cortex, TP, and amygdala causes similar socioemotional deficits. To our knowledge, ours is the first study showing gray matter atrophy in the temporal pole in regular cannabis users and the degree of atrophy related to the frequency of drug use in the 3 months preceding inclusion in the study. Previous research using ROI analysis did not focus on possible changes in this region. With regard to the two studies using VBM, one (Matochik et al, 2005) found changes only in the medial part of the temporal cortex, maybe due to the small sample studied; the other (Cousijn et al, 2012) failed to find any change in the cerebral cortex. Atrophy of this structure has been found in cocaine users (AlbeinUrios et al, 2013) and has been linked to socioemotional and personality problems.

In addition to the changes in the polar regions, there are also changes in the medial temporal cortex, which is one of the structures often reported to be associated with cannabis addiction and where we find a strong bilateral decrease in gray matter volume in the population of regular cannabis users. Such a pattern of atrophy has been also described in other forms of addiction such as alcohol addiction (Mechtcheriakov et al, 2007), but not in heroin users (Denier et al, 2013). However, other patients with severe, non-toxic, behavioral addiction such as pathological gambling (Levine et al, 2005) do not present the same form of atrophy, suggesting that temporal atrophy is indeed associated with cannabis consumption rather than with addictive behavior itself. Despite the fact that the neurobiological interpretation of this volume reduction is still unclear, studies on rodents give some clues on this point.
Scallet et al (1987) found a THC-induced decrease in the mean volume of hippocampal neurons and a $44 \%$ reduction in the number of synapses up to 7 months after exposure. Functional and structural variations in the hippocampus have been linked to reduced memory performance (Solowij and Battisti, 2008) and psychotic symptoms (Yücel et al, 2008). Cannabis exposure produces reduced activation in the hippocampus during verbal and visual learning tasks (Block et al, 2002; Jager et al, 2007).

Another main finding of our study is the increase in gray matter volume in the cerebellum that replicates results in adults (Cousijn et al, 2012) and in adolescents (Medina et al, 2010).

In normal adolescents the volume of cerebellar gray matter starts to decrease around puberty and continues until early adulthood (Diamond, 2000; Ostby et al, 2009). Alterations of this phenomenon have been observed in various psychiatric conditions (Mackie et al, 2007) (Jarvis et al, 2008) (Castellanos et al, 2002) (Pujol et al, 2004) (Hill et al, 2003) and in adolescents with familiar history of severe alcohol abuse (Hill et al, 2007).

It has been hypothesized that this normal reduction in gray matter volume in the cerebellum is due to the pruning of the synaptic connections (Cohen-Cory, 2002). One possible reason for abnormal pruning could be the toxic effect of THC at a critical period of brain maturation. Endogenous cannabinoids have an important role in synaptic pruning due to their interaction with GB1 receptors controlling the release of glutamate and GABA (Bossong and Niesink, 2010). Exogenous cannabinoids might disturb this system by competing for the receptors, thus inhibiting the pruning particularly in receptorrich areas like the cerebellum (Casu et al, 2005) or the prefrontal cortex (Bossong and Niesink, 2010).

However, our results cannot exclude that abnormal pruning is due to genetic predisposition as seen in children from multiplex alcoholic families even before the beginning of any drinking behavior or in obsessive compulsive disorder (Hill et al, 2007). Hence, a limitation of our study and a matter of debate in the literature are connected to the question of whether these brain changes are caused by cannabis use or are already present before drug use.

Additional limitation of our study is the relatively narrow age-range of our participants (19-29 years of age) that may limit the possibility to totally capture the cumulative effects of cannabis. On the other hand, a 10-year window of age range assures the homogeneity of the two groups, aspect of paramount importance in the context of group analysis.

Existing literature shows that cognitive alterations and $\mathrm{CB} 1$ receptor downregulation in regular cannabis users may return to normal values due to neuroadaptive phenomena occurring after periods of abstinence (Bosker et al, 2013; Hanson et al, 2010; Hirvonen et al, 2012; Schweinsburg et al, 2010). The design of our study cannot address whether the structural alterations observed are permanent or reversible. The so far unexplored evolution of the gray matter alterations across time and the possible recovery after recency of use should be addressed by further longitudinal studies.

In our study, we provide new arguments about the effects of long-term exposure to cannabis on brain structure integrity. We were able to support all the hypotheses raised in the introduction: (i) we demonstrate that regular cannabis use is 
associated with reduced gray matter volume in regions rich in cannabinoid CB1 receptors that are functionally linked to motivational, emotional, and affective processing. (ii) We complete our findings by showing that the magnitude of changes in these regions correlates with the frequency of cannabis use and (iii) is modulated by the age at which consumption was initiated.

We present a different scenario in the cerebellum where the increase in gray matter volume in regular users without any correlation with the amount of cannabis use may have a developmental nature. The line of research should move toward longitudinal studies in order to differentiate between consumption-related and developmental aspects of brain changes associated with long-term regular cannabis exposure.

\section{FUNDING AND DISCLOSURE}

This project was supported by the Swiss National Scientific Research Foundation (grant SNF 320030_127507/1) and the Faculty of Biology and Medicine of the University of Lausanne (multidisciplinary project FBM). The authors declare no conflict of interest.

\section{ACKNOWLEDGEMENTS}

The work was supported by the Centre d'Imagerie BioMédicale (CIBM) of the University of Lausanne (UNIL), the Swiss Federal Institute of Technology Lausanne (EPFL), the University of Geneva (UniGe), the Centre Hospitalier Universitaire Vaudois (CHUV), the Hôpitaux Universitaires de Genève (HUG), and the Leenaards and Jeantet Foundations. We thank Professor Patrice Mangin, Dr Christian Staub, Dr Marc Augsburger of the University Center of Forensic Medicine Lausanne-Geneva, Professor Thierry Buclin, Monique Appenzeller, and the staff of the division of Clinical Pharmacology and Toxicology at CHUV. We also thank Ms Dasha Polzik (University of Chicago) for her assistance in preparing the manuscript.

\section{REFERENCES}

Albein-Urios N, Martinez-Gonzalez JM, Lozano O, Moreno-López L, Soriano-Mas C, Verdejo-Garcia A (2013). Negative urgency, disinhibition and reduced temporal pole gray matter characterize the comorbidity of cocaine dependence and personality disorders. Drug Alcohol Depend 132: 231-237.

Ashburner J (2007). A fast diffeomorphic image registration algorithm. Neuroimage 38: 95-113.

Ashburner J, Friston KJ (2005). Unified segmentation. Neuroimage 26: 839-851.

Ashtari M, Avants B, Cyckowski L, Cervellione KL, Roofeh D, Cook P et al (2011). Medial temporal structures and memory functions in adolescents with heavy cannabis use. J Psychiatr Res 45: 1055-1066.

Batalla A, Bhattacharyya S, Yücel M, Fusar-Poli P, Crippa JA, Nogué $S$ et al (2013). Structural and functional imaging studies in chronic cannabis users: a systematic review of adolescent and adult findings. PLOS ONE 8: e55821.

Battistella G, Fornari E, Thomas A, Mall JF, Chtioui H, Appenzeller M et al (2013). Weed or wheel! FMRI, behavioural, and toxicological investigations of how cannabis smoking affects skills necessary for driving. PLoS ONE 8: e52545.

Bechara A, Damasio H (2002). Decision-making and addiction (part I): impaired activation of somatic states in substance dependent individuals when pondering decisions with negative future consequences. Neuropsychologia 40: 1675-1689.

Block RI, O'Leary DS, Hichwa RD, Augustinack JC, Boles Ponto LL, Ghoneim MM et al (2002). Effects of frequent marijuana use on memory-related regional cerebral blood flow. Pharmacol Biochem Behav 72: 237-250.

Bosker WM, Karschner EL, Lee D, Goodwin RS, Hirvonen J, Innis RB et al (2013). Psychomotor function in chronic daily Cannabis smokers during sustained abstinence. PLoS ONE 8: e53127.

Bossong MG, Niesink RJ (2010). Adolescent brain maturation, the endogenous cannabinoid system and the neurobiology of cannabis-induced schizophrenia. Prog Neurobiol 92: 370-385.

Burns HD, Van Laere K, Sanabria-Bohórquez S, Hamill TG, Bormans G, Eng W-s et al (2007). [18F]MK-9470, a positron emission tomography (PET) tracer for in vivo human PET brain imaging of the cannabinoid-1 receptor. Proc Natl Acad Sci USA 104: 9800-9805.

Castellanos FX, Lee PP, Sharp W, Jeffries NO, Greenstein DK, Clasen LS et al (2002). Developmental trajectories of brain volume abnormalities in children and adolescents with attention-deficit/hyperactivity disorder. JAMA 288: 1740-1748.

Casu MA, Pisu C, Sanna A, Tambaro S, Spada GP, Mongeau R et al (2005). Effect of delta9-tetrahydrocannabinol on phosphorylated CREB in rat cerebellum: an immunohistochemical study. Brain Res 1048: 41-47.

Churchwell JC, Lopez-Larson M, Yurgelun-Todd DA (2010). Altered frontal cortical volume and decision making in adolescent cannabis users. Front Psychol 1: 225.

Cohen JA (1988). Statistical Power Analysis for the Behavioral Sciences (2nd Ed.) Lawrence Erlbaum Associates, Hillsdale, NJ, USA.

Cohen-Cory S (2002). The developing synapse: construction and modulation of synaptic structures and circuits. Science 298: 770-776.

Connolly CG, Bell RP, Foxe JJ, Garavan H (2013). Dissociated grey matter changes with prolonged addiction and extended abstinence in cocaine users. PLoS ONE 8: e59645.

Cousijn J, Wiers RW, Ridderinkhof KR, van den Brink W, Veltman DJ, Goudriaan AE (2012). Grey matter alterations associated with cannabis use: results of a VBM study in heavy cannabis users and healthy controls. Neuroimage 59: 3845-3851.

Degenhardt L, Chiu WT, Sampson N, Kessler RC, Anthony JC, Angermeyer $\mathrm{M}$ et al (2008). Toward a global view of alcohol, tobacco, cannabis, and cocaine use: findings from the WHO World Mental Health Surveys. PLoS Med 5: e141.

Demirakca T, Sartorius A, Ende G, Meyer N, Welzel H, Skopp G et al (2011). Diminished gray matter in the hippocampus of cannabis users: possible protective effects of cannabidiol. Drug Alcohol Depend 114: 242-245.

Denier N, Schmidt A, Gerber H, Schmid O, Riecher-Rossler A, Wiesbeck GA et al (2013). Association of frontal gray matter volume and cerebral perfusion in heroin addiction: a multimodal neuroimaging study. Front Psychiatry 4: 135.

Diamond A (2000). Close interrelation of motor development and cognitive development and of the cerebellum and prefrontal cortex. Child Dev 71: 44-56.

Downer E, Boland B, Fogarty M, Campbell V (2001). Delta 9tetrahydrocannabinol induces the apoptotic pathway in cultured cortical neurones via activation of the CB1 receptor. Neuroreport 12: 3973-3978.

Draganski B, Bhatia KP (2010). Brain structure in movement disorders: a neuroimaging perspective. Curr Opin Neurol 23: 413-419.

Fabritius M, Chtioui H, Battistella G, Annoni JM, Dao K, Favrat B et al (2013a). Comparison of cannabinoid concentrations in oral fluid and whole blood between occasional and regular cannabis smokers prior to and after smoking a cannabis joint. Anal Bioanal Chem 405: 9791-9803. 
Fabritius M, Favrat B, Chtioui H, Battistella G, Annoni JM, Appenzeller $\mathrm{M}$ et al (2013b). THCCOOH concentrations in whole blood: Are they useful in discriminating occasional from heavy smokers? Drug Test Anal 6: 155-163.

Good CD, Ashburner J, Frackowiak RS (2001). Computational neuroanatomy: new perspectives for neuroradiology. Rev Neurol 157(8-9 Pt 1): 797-806.

Gruber SA, Silveri MM, Dahlgren MK, Yurgelun-Todd D (2011). Why so impulsive? White matter alterations are associated with impulsivity in chronic marijuana smokers. Exp Clin Psychopharmacol 19: 231-242.

Hanson KL, Winward JL, Schweinsburg AD, Medina KL, Brown SA, Tapert SF (2010). Longitudinal study of cognition among adolescent marijuana users over three weeks of abstinence. Addict Behav 35: 970-976.

Hill JM, Ades AM, McCune SK, Sahir N, Moody EM, Abebe DT et al (2003). Vasoactive intestinal peptide in the brain of a mouse model for Down syndrome. Exp Neurol 183: 56-65.

Hill SY, Muddasani S, Prasad K, Nutche J, Steinhauer SR, Scanlon J et al (2007). Cerebellar volume in offspring from multiplex alcohol dependence families. Biol Psychiatry 61: 41-47.

Hirvonen J, Goodwin RS, Li CT, Terry GE, Zoghbi SS, Morse C et al (2012). Reversible and regionally selective downregulation of brain cannabinoid CB1 receptors in chronic daily cannabis smokers. Mol Psychiatry 17: 642-649.

Hurd YL, Michaelides M, Miller ML, Jutras-Aswad D (2013). Trajectory of adolescent cannabis use on addiction vulnerability. Neuropharmacology 76(Pt B): 416-424.

Jager G, Van Hell HH, De Win MML, Kahn RS, Van Den Brink W, Van Ree JM et al (2007). Effects of frequent cannabis use on hippocampal activity during an associative memory task. Eur Neuropsychopharmacol 17: 289-297.

Jarvis K, DelBello MP, Mills N, Elman I, Strakowski SM, Adler CM (2008). Neuroanatomic comparison of bipolar adolescents with and without cannabis use disorders. J Child Adolesc Psychopharmacol 18: 557-563.

Kling A, Steklis HD (1976). A neural substrate for affiliative behavior in nonhuman primates. Brain Behav Evol 13: 216-238.

Koob GF, Volkow ND (2010). Neurocircuitry of addiction. Neuropsychopharmacology 35: 217-238.

Lawston J, Borella A, Robinson JK, Whitaker-Azmitia PM (2000). Changes in hippocampal morphology following chronic treatment with the synthetic cannabinoid WIN 55,212-2. Brain Res 877: 407-410.

Levine B, Black SE, Cheung G, Campbell A, O’Toole C, Schwartz ML (2005). Gambling task performance in traumatic brain injury: relationships to injury severity, atrophy, lesion location, and cognitive and psychosocial outcome. Cogn Behav Neurol 18: 45-54.

Lorenzetti V, Lubman DI, Whittle S, Solowij N, Yücel M (2010). Structural MRI findings in long-term cannabis users: what do we know? Subst Use Misuse 45: 1787-1808.

Mackie S, Shaw P, Lenroot R, Pierson R, Greenstein DK, Nugent TF 3rd et al (2007). Cerebellar development and clinical outcome in attention deficit hyperactivity disorder. Am J Psychiatry 164: 647-655.

Matochik JA, Eldreth DA, Cadet J-L, Bolla KI (2005). Altered brain tissue composition in heavy marijuana users. Drug Alcohol Depend 77: 23-30.

Mechtcheriakov S, Brenneis C, Egger K, Koppelstaetter F, Schocke M, Marksteiner J (2007). A widespread distinct pattern of cerebral atrophy in patients with alcohol addiction revealed by voxel-based morphometry. J Neurol Neurosurg Psychiatry 78: 610-614.

Medina KL, Nagel BJ, Tapert SF (2010). Abnormal cerebellar morphometry in abstinent adolescent marijuana users. Psychiatry Res 182: 152-159.

Olson IR, Plotzker A, Ezzyat Y (2007). The Enigmatic temporal pole: a review of findings on social and emotional processing. Brain 130(Pt 7): 1718-1731.

Ostby Y, Tamnes CK, Fjell AM, Westlye LT, Due-Tonnessen P, Walhovd KB (2009). Heterogeneity in subcortical brain development: a structural magnetic resonance imaging study of brain maturation from 8 to 30 years. J Neurosci 29: 11772-11782.

Paus T, Keshavan M, Giedd JN (2008). Why do many psychiatric disorders emerge during adolescence? Nat Rev Neurosci 9: 947-957.

Paus T, Zijdenbos A, Worsley K, Collins DL, Blumenthal J, Giedd JN et al (1999). Structural maturation of neural pathways in children and adolescents: in vivo study. Science 283: 1908-1911.

Pujol J, Soriano-Mas C, Alonso P, Cardoner N, Menchon JM, Deus J et al (2004). Mapping structural brain alterations in obsessive-compulsive disorder. Arch Gen Psychiatry 61: 720-730.

Scallet AC, Uemura E, Andrews A, Ali SF, McMillan DE, Paule MG et al (1987). Morphometric studies of the rat hippocampus following chronic delta-9-tetrahydrocannabinol (THC). Brain Res 436: 193-198.

Schweinsburg AD, Schweinsburg BC, Medina KL, McQueeny T, Brown SA, Tapert SF (2010). The influence of recency of use on fMRI response during spatial working memory in adolescent marijuana users. J Psychoactive Drugs 42: 401-412.

Seeley WW, Menon V, Schatzberg AF, Keller J, Glover GH, Kenna H et al (2007). Dissociable intrinsic connectivity networks for salience processing and executive control. J Neurosci 27: 2349-2356.

Solowij N, Battisti R (2008). The chronic effects of cannabis on memory in humans: a review. Curr Drug Abuse Rev 1: 81-98.

Sullivan EV, Müller-Oehring E, Pitel A-L, Chanraud S, Shankaranarayanan A, Alsop DC et al (2013). A selective insular perfusion deficit contributes to compromised salience network connectivity in recovering alcoholic men. Biol Psychiatry 74: 547-555.

Thompson SA, Patterson K, Hodges JR (2003). Left/right asymmetry of atrophy in semantic dementia: behavioral-cognitive implications. Neurology 61: 1196-1203.

Vaidya JG, Block RI, O'Leary DS, Ponto LB, Ghoneim MM, Bechara A (2012). Effects of chronic marijuana use on brain activity during monetary decision-making. Neuropsychopharmacology 37: 618-629.

Wiers RW, Bartholow BD, van den Wildenberg E, Thush C, Engels RC, Sher KJ et al (2007). Automatic and controlled processes and the development of addictive behaviors in adolescents: a review and a model. Pharmacol Biochem Behav 86: 263-283.

Yücel M, Solowij N, Respondek C, Whittle S, Fornito A, Pantelis C et al (2008). Regional brain abnormalities associated with longterm heavy cannabis use. Arch Gen Psychiatry 65: 694-701.

Zalesky A, Solowij N, Yucel M, Lubman DI, Takagi M, Harding IH et al (2012). Effect of long-term cannabis use on axonal fibre connectivity. Brain 135(Pt 7): 2245-2255.

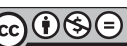

This work is licensed under a Creative Commons Attribution-NonCommercial-NoDerivs 3.0 Unported License. To view a copy of this license, visit http:// creativecommons.org/licenses/by-nc-nd/3.0/

Supplementary Information accompanies the paper on the Neuropsychopharmacology website (http://www.nature.com/npp) 